\title{
Paleolithic ketogenic diet (PKD) in chronic diseases: Clinical and research data
}

\author{
Zsófia Clemens, Csaba Tóth
}

International Center for Medical Nutritional Intervention (ICMNI) - Paleomedicina Hungary

Email: clemenszsofia@gmail.com; tothcsaba.pk30@gmail.com

In the presentation we give an overview of our clinical experience and research with the paleolithic ketogenic diet (PKD), a diet that was developed by the ICMNI team. Since 2012 we have been exclusively using this approach in the treatment of a wide array of chronic conditions. We use the combined term "paleolithic ketogenic" because the diet has its roots in both, the paleolithic and the ketogenic diet. The PKD combines the benefits of the two diets while excluding shortcomings of both diets. We have to emphasise the fact that both the classic version of the ketogenic diet and the popular version of the paleolithic diet are associated with side-effects. The side-effects of the classic ketogenic diet have been welldocumented through its use over the last hundred years, whereas, the shortcomings of the popular paleolithic diet are less well-known. As examples for the side-effects, we present a patient who, after 7 years on the popular paleolithic diet, developed severe atherosclerosis, and another patient who, after following the classic ketogenic diet for 17 years, developed tophi (deposition of urate crystals in soft tissues) in multiple locations on hands and feet. Since 2012 we have been using the PKD on more than 4000 patients including those with autoimmune diseases, cancer, psychiatric and neurologic conditions. In the presentation we focus on presenting data on type 1 diabetes (T1DM) and cancer patients, as well as showing research data related to intestinal permeability measurements in healthy persons as well as in patients.

\section{Type 1 diabetes mellitus (T1DM)}

Three major benefits are known to be associated with low carb (or ketogenic) diets: (1) glucose control, (2) decreasing insulin need and (3) decreasing the number of hypoglycemic episodes [1]. The PKD confers the same benefits in T1DM but in addition, it also ensures inflammation control [2,3] which is of utmost importance given the autoimmune origin of the disease. By controlling inflammation, the PKD likely prevents the development of long-term complications [4]. Moreover, in patients with new-onset T1DM, intervening with the PKD also results in preventing further decay of their own insulin production as shown by stable or even increased C-peptide measurements following a shift toward the PKD [2,3]. In the presentation we show six cases with T1DM where normoglycemia and long-term insulin freedom was achieved, along with preserved C-peptide production. We emphasize that measuring C-peptide in any low-carb diet may be misleading with regard to the assessement of the patient's own insulin production capacity [3]. In order to differentiate the effect of a low-carb diet and the compromised insulin capacity itself, as a new tool, we have introduced the measurement of the stimulated C-peptide in addition to the regular fasting C-peptide. 


\section{T1DM: C-PEPTIDE}

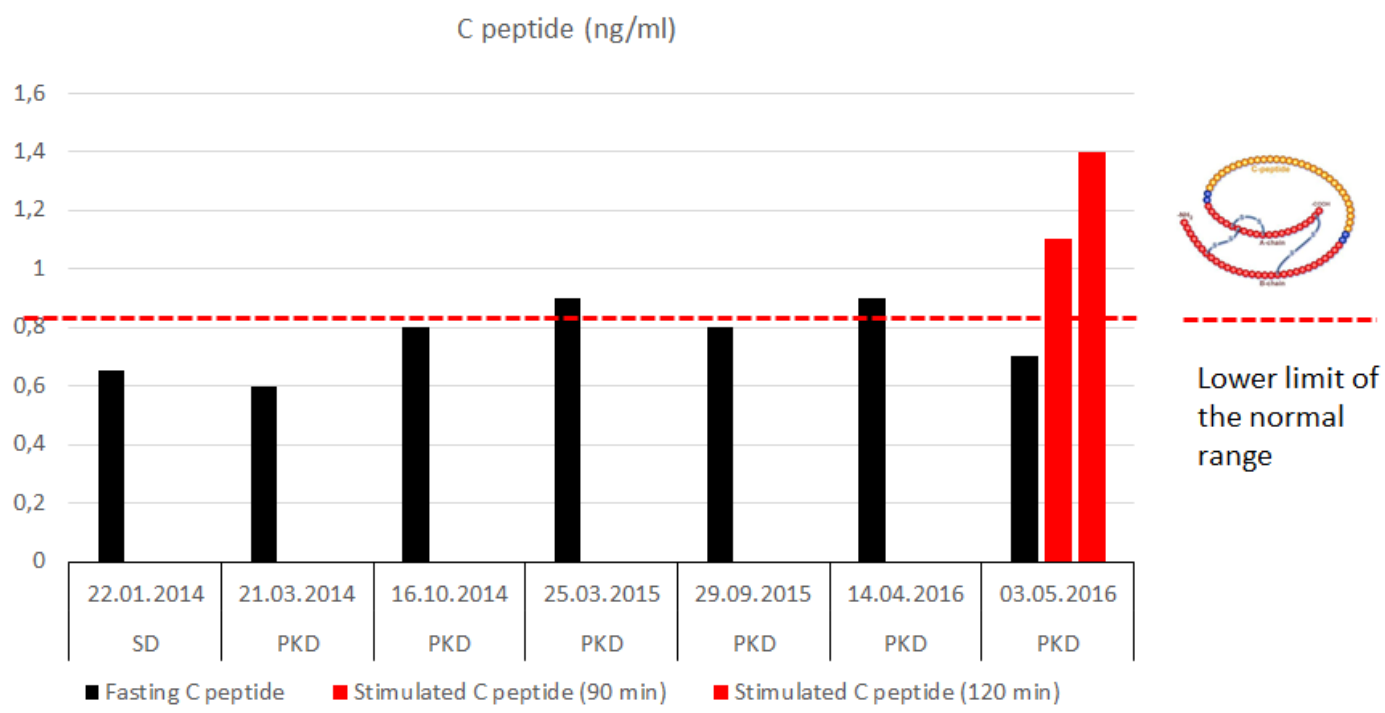

20/M, Follow-up: 3 years on the
paleolithic ketogenic diet (PKD)

Figure 1

C-peptide in a T1DM patient following the PKD for three years. Note that C-peptide was preserved during follow-up. C-peptide after stimulation was in the normal range.

\section{Cancer}

We present our cancer-PKD database including 47 patients. An assessment of the database shows that out of all patients, long-term survivors are those who highly adhere to the PKD, and along with this, denied chemotherapy and radiotherapy. We highlight the case of a glioblastoma patient who has been on the PKD as a stand-alone therapy, and is still progression-free and symptom-free at 25 months on the PKD, and 32 month after diagnosis onset. Another glioblastoma patient who also strictly adhered to the PKD, but in addition used radiotherapy as well as alternative treatments including HBOT and supplements, died at 11 months on the diet.

\begin{tabular}{|c|c|c|c|c|c|c|c|c|}
\hline Still living & $\begin{array}{c}\text { Currently } \\
\text { on the } \\
\text { diet } \\
(09.2018)\end{array}$ & $\begin{array}{c}\text { Diet } \\
\text { duration } \\
\text { (months) }\end{array}$ & $\begin{array}{c}\text { Tendency while on } \\
\text { the PKD }\end{array}$ & Diet adherence & Dignosis & Chemotherapy & Radiotherapy & Surgery \\
\hline YES & YES & 24 & Stable & High & Glioblastoma, recurrent & Yes (previously) & Yes (previously) & Yes (previously) \\
\hline YES & YES & 35 & Regression & High & $\mathrm{CIN}$, recurrent & No & No & Yes (previously) \\
\hline YES & YES & 83 & No change & High & Colon cancer, grade 1 & No & No & No \\
\hline YES & YES & 45 & Stable & High & Soft palate tumor & No & No & No \\
\hline YES & YES & 53 & Stable_progr_regr & High_lower_high & Brain tumor, recurrent & No & No & Yes (previously) \\
\hline YES & YES & 63 & Stable & High & Breast cancer & No & No & No \\
\hline
\end{tabular}

\section{Table 1.}

Cancer patients who outperformed their diagnosis not just by being alive but also by being progression-free. A temporary decrease in the PKD adherence in a patient with recurrent brain tumor, was associated with slight progression, while going back to strictly following the diet was associated with regression. Note that none of the patients used radiotherapy and chemotherapy along with the diet therapy and only one patient had radiochemotherapy before starting the diet therapy. 


\section{Intestinal permeability (IP) measurements}

We use the PEG400 challenge test to measure IP [5]. The PEG400 has many benefits over other IP tests. Since 2017 we have been measuring IP in our own laboratory in collaboration with the Department of Applied Biotechnology and Food Science, University of Technology and Economy, Budapest. Measurements show that following the PKD is associated with normal IP, and that originally elevated IP (associated either with the Western-type diet, the classic ketogenic diet or the paleolithic diet) normalizes after a shift toward the PKD in both healthy persons and patients. Taking medicines or supplements can also be associated with elevated IP.

\section{PEG400 CHALLENGE TEST}

in a patient with Hodgkin lymphoma

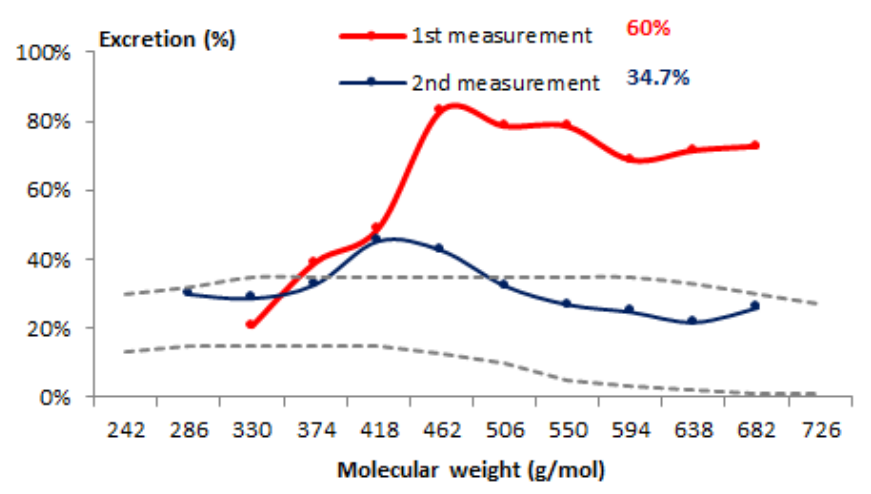

Figure 3.

PEG400 test result in a patient with Hodgkin lymphoma. The patient was originally following the classic ketogenic diet with multiple supplements. The first measurement was done right before PKD onset (and after several cycles of chemotherapy regimens). The second measurement was done at 3.5 months on the PKD without chemotherapy. Normalization of the intestinal permeability was paralleled by symptomatic improvement.

\section{References}

[1] Nielsen JV, Gando C, Joensson E, Paulsson C. Low carbohydrate diet in type 1 diabetes, long-term improvement and adherence: A clinical audit. Diabetol Metab Syndr 2012;4:23. doi:10.1186/1758-5996-4-23.

[2] Tóth C, Clemens Z. Type 1 diabetes mellitus successfully managed with the paleolithic ketogenic diet. Int J Case Reports Images 2014;5:699-703. doi:10.5348/ijcri-2014124CR-10435.

[3] Tóth C, Clemens Z. A child with type 1 diabetes mellitus (T1DM) successfully treated with the Paleolithic ketogenic diet: A 19-month insulin-freedom. Int J Case Reports Images 2015;6:752-7. doi:10.5348/ijcri-2015121-CR-10582.

[4] Ziegler D, Behler M, Schroers-Teuber M, Roden M. Near-normoglycaemia and development of neuropathy: A 24-year prospective study from diagnosis of type 1 diabetes. BMJ Open 2015;5:e006559. doi:10.1136/bmjopen-2014-006559.

[5] Chadwick VS, Phillips SF, Hofmann AF. Measurements of intestinal permeability using low molecular weight polyethylene glycols (PEG 400). I. Chemical analysis and biological properties of PEG 400. Gastroenterology 1977;73:241-6. 